\title{
Muda vocal: el proceso de cambio de la voz durante la pubertad
}

\author{
Vocal mutation: the voice change process during puberty
}

\author{
Loreto Nercelles, ${ }^{*,+}$ Diana Centeno ${ }^{\ddagger}$ \\ * Fonoaudiología, Universidad Andrés Bello, ${ }^{\ddagger}$ Departamento de Otorrinolaringología, \\ Hospital de Niños “Dr. Luis Calvo Mackenna”, Santiago, Chile.
}

\begin{abstract}
RESUMEN
Introducción: La muda vocal es el periodo de cambio de la voz que ocurre durante la pubertad. Se caracteriza por modificaciones acústicas e inestabilidades vocales que ocurre tanto en varones como en mujeres. Objetivo: Realizar una revisión narrativa de estudios publicados sobre muda vocal, ya sea de voz hablada o cantada. Se llevó a cabo la búsqueda de artículos publicados entre 1990 a 2019 en PubMed y LILACS. Resultados: la revisión bibliográfica constó de ocho artículos. Los estudios revisados incluyeron de seis a 600 sujetos. Las investigaciones se realizaron en hombres y mujeres tanto en voz hablada como cantada. En general, los datos de los estudios revelaron que la edad de muda vocal es después de los 13 años y que puede haber repercusiones emocionales durante ese periodo. Conclusión: Existe poca literatura que investigue acerca de los cambios de la voz en la pubertad. La muda vocal implica diversas modificaciones en los aspectos orgánico, funcional y emocional del individuo.
\end{abstract}

Palabras clave: Voz, disfonía, cambio de la voz, muda vocal, adolescentes.

\section{INTRODUCCIÓN}

La voz sufre innumerables modificaciones durante el transcurso de la vida; sin embargo, durante la pubertad se produce un cambio más notorio, llamado periodo de la muda vocal. Entre las características de esta etapa

\footnotetext{
+ Correspondencia: LN, Inercelles@gmail.com

Conflicto de intereses: Las autoras declaran que no tienen. Citar como: Nercelles L, Centeno D. Muda vocal: el proceso de cambio de la voz durante la pubertad. Rev Mex Pediatr 2020; 87(4): 153-157. https://dx.doi.org/10.35366/95827
}

\begin{abstract}
Introduction: The vocal mutation is the period of voice change that occurs during puberty. It is characterized by acoustic modifications and vocal instabilities that occur in both men and women. Objective: To conduct a narrative review of published studies on vocal mutation, whether spoken or sung. A search of papers published between 1990 and 2019 in PubMed and LILACS was performed. Results: the bibliographic review consisted of eight articles. The studies reviewed included from six to 600 subjects. The investigations were carried out in men and women in both spoken and sung voices. Overall, study data revealed that vocal mutation occurs after age 13, and that emotional repercussions may occur during this period. Conclusion: To date, there are few published studies on changes in the voice in puberty. The vocal mutation involves various modifications in the organic, functional and emotional aspects of the individual.
\end{abstract}

Keywords: Voice, dysphonia, voice change, vocal mutation, adolescents.

destacan que es más evidente en varones y se reduce la frecuencia fundamental, predominando el registro de pecho. Además, existe inestabilidad en la emisión, diplofonía, aspereza y soplosidad. ${ }^{1}$

El periodo de muda vocal representa una etapa de desequilibrios, caracterizada por modificaciones anatómicas, tales como aumento de longitud del cuello, descenso de la laringe, crecimiento de la epiglotis y de la glándula tiroides, ensanchamiento del tórax, crecimiento de las cavidades de resonancia, de la tráquea y los pulmones..$^{2-6}$

Existe controversia entre los autores en relación con la edad de inicio de la muda vocal. Algunos la definen 
como un cambio en la voz que se manifiesta alrededor de los 12 años de edad. No obstante, otros sostienen que el cambio de voz en varones se produce de los 13 a los 15 años, mientras que en las mujeres ocurre de los 12 a los 14 años. ${ }^{3,7}$

La estabilización de la muda vocal podría durar de tres a ocho meses en la voz hablada, , ${ }^{4-10}$ y en relación con la voz cantada demoraría uno o dos años en estabilizarse. ${ }^{10,11}$ Los expertos en voz cantada aseguran que, durante la etapa de muda, la voz puede seguir entrenándose, por lo que no es necesario abandonar el canto, sino adaptarlo. ${ }^{12}$

Durante todo el periodo de la muda, en el varón se observa un descenso de un total de una octava, mientras que en las mujeres es de alrededor de una tercera. Si bien parece ser que los cambios obedecen a la morfología diferente, es posible que los valores que se obtengan en la tesitura dependan también del entrenamiento vocal que tenga cada sujeto. ${ }^{13}$

El proceso de muda vocal no siempre se realiza con normalidad. Hay ocasiones en que el cambio de voz se retrasa o no se completa, persistiendo una voz infantil, ${ }^{14}$ lo cual se denomina trastornos de la muda vocal o puberfonías. La sintomatología principal es la frecuencia más aguda a la correspondiente a la edad y el sexo. Además, pueden existir bitonalidades, respiración y coordinación fonorrespiratoria, con posibles alteraciones y aumento de tensión en la zona perilaríngea generada por el esfuerzo de mantener la laringe en posición alta. ${ }^{2,6,8,10,15-17}$ Las causas son múltiples pero generalmente son problemas de adaptación muscular, ambientales o psicosociales. ${ }^{6,7,18-20}$

Objetivo: realizar una síntesis de las investigaciones sobre la etapa de la muda vocal para actualizar a la comunidad médica sobre este tema.

\section{MATERIAL Y MÉTODOS}

Se realizó una búsqueda de la literatura de artículos publicados entre los años 1990 a 2019 en las bases de datos PubMed y LILACS

Para la localización de las publicaciones se usaron como palabras clave: muda vocal y mutación vocal, en español, inglés y portugués.

Se excluyeron artículos de revisión, los que no presentaban resumen, conclusiones o los resultados de la investigación. También los que se referían al trastorno de la muda vocal en toda su extensión y no al proceso de muda vocal. De un total de 19 artículos, el número final de artículos seleccionados fue de ocho investigaciones.

\section{RESULTADOS}

En las investigaciones revisadas se incluyeron entre seis a 600 sujetos, en las cuales se incluyeron hombres y mujeres. De los estudios revisados, dos corresponden a voz cantada y seis en voz hablada.

La Tabla 1 resume los principales aspectos de los artículos encontrados separados por autor, año, artículo, objetivo, muestra, metodología y resultados.

\section{INICIO DE LA MUDA VOCAL Y CARACTERÍSTICAS VOCALES}

Existen varias investigaciones que intentan predecir el inicio de la muda vocal, en especial de voz cantada. Fuchs y colaboradores indican que los valores de testosterona y la velocidad de crecimiento parecen ser los factores que más se correlacionan con el inicio de muda vocal. ${ }^{8}$

Por su parte, Saida y colaboradores indican que el crecimiento físico y el cambio de la voz asociado con la etapa de la pubertad no ocurren en forma simultánea. Dentro de la etapa de pubertad los autores refieren fases rápidas y lentas. ${ }^{23}$

En relación con la edad de inicio, Chernobelsky encuentra voz más agravada en niños entre 12 y 13 años en comparación a niños de 11 a 12 años. ${ }^{22}$ Sobre esta misma valoración, Balasubramaniam encuentra diferencias significativas en la frecuencia fundamental de la voz entre voces masculinas y femeninas a los 14 años de edad. ${ }^{23}$

\section{SENTIMIENTOS ASOCIADOS EN EL PERIODO DE MUDA VOCAL}

Esta etapa se describe como un periodo lleno de cambios y desequilibrios; por lo anterior, es de esperar que sea algo notorio, especialmente dentro de la población masculina, quienes modifican su voz en mayor grado que las mujeres. El estudio de Damasceno y colaboradores reporta que los varones se sienten incómodos en esta etapa y que el cambio de voz es un factor negativo en la interacción con los interlocutores. ${ }^{24}$ Sin embargo, en el estudio retrospectivo de Gil y su equipo, quienes entrevistaron a hombres que ya habían superado la etapa de muda vocal, si bien $78.8 \%$ de los varones notó cambios de voz durante la pubertad, no encontraron sentimientos negativos. ${ }^{25}$

Una interesante experiencia es la que se plantea en el proyecto Young Doctor, en el que se realizan planes educativos para enseñar a jóvenes sobre 
Tabla 1: Características de los estudios incluidos en la revisión.

\begin{tabular}{|c|c|c|c|c|}
\hline $\begin{array}{l}\text { Autores / } \\
\text { Año / País }\end{array}$ & Objetivo & Muestra & Metodología & Resultados \\
\hline $\begin{array}{l}\text { Saida H y } \\
\text { col. }{ }^{21} \\
\text { / Japón/ } \\
1990\end{array}$ & $\begin{array}{l}\text { Observar la relación entre } \\
\text { la mutación de la voz y el } \\
\text { crecimiento corporal }\end{array}$ & $\begin{array}{l}100 \text { estudiantes varones } \\
\text { en su pubertad }\end{array}$ & $\begin{array}{l}\text { Estudio longitudinal. Se realizaron } \\
\text { grabaciones de voz dos veces, } \\
\text { anualmente. Se obtuvo la frecuencia } \\
\text { fundamental (F0) y los valores } \\
\text { formantes }\end{array}$ & $\begin{array}{l}\text { 1. Hubo correlación negativa en } \\
\text { la tasa de cambio entre F0 y el } \\
\text { cambio en peso y estatura } \\
\text { 2. El periodo de mutación consistía } \\
\text { en fases rápida y lenta } \\
\text { 3. Los cambios en la voz ocurrieron } \\
\text { posterior al crecimiento físico y al de- } \\
\text { sarrollo de la prominencia laríngea }\end{array}$ \\
\hline $\begin{array}{l}\text { Gil D y col. }{ }^{25} \\
\text { / Brasil / } \\
1994\end{array}$ & $\begin{array}{l}\text { Describir los sentimientos } \\
\text { negativos asociados al } \\
\text { periodo de muda vocal }\end{array}$ & $\begin{array}{l}400 \text { hombres postmuda } \\
\text { vocal }\end{array}$ & $\begin{array}{l}\text { Aplicación de un cuestionario con } \\
\text { preguntas sobre sentimientos } \\
\text { negativos relacionados con la etapa } \\
\text { de muda vocal }\end{array}$ & $\begin{array}{l}\text { 1. El } 78 \% \text { notó cambios de la voz } \\
\text { durante la pubertad. Los cambios } \\
\text { más observados fueron inestabili- } \\
\text { dad, voz desafinada y ronca } \\
\text { 2. En general, los entrevistados no } \\
\text { recordaron sentimientos negativos } \\
\text { durante etapa de muda vocal }\end{array}$ \\
\hline $\begin{array}{l}\text { Fuchs M y } \\
\text { col. }{ }^{8} \\
\text { / Alemania } \\
\text { /1999 }\end{array}$ & $\begin{array}{l}\text { Identificar si existe algún } \\
\text { factor predictivo del inicio } \\
\text { de la mutación vocal }\end{array}$ & 36 miembros de un coro & $\begin{array}{l}\text { Se midieron } 10 \text { parámetros vocales } \\
\text { y ocho no vocales cada tres meses, } \\
\text { durante un periodo de } 3.5 \text { años y se } \\
\text { evaluó su valor predictivo }\end{array}$ & $\begin{array}{l}\text { La predicción más precisa de la } \\
\text { mutación de la voz fue dada por el } \\
\text { nivel de testosterona y la velocidad } \\
\text { del crecimiento }\end{array}$ \\
\hline $\begin{array}{l}\text { Santos M y } \\
\text { col. }{ }^{27} \\
\text { / Brasil / } \\
2007\end{array}$ & $\begin{array}{l}\text { Evaluar la progresión de } \\
\text { las lesiones estructurales } \\
\text { de las cuerdas vocales, } \\
\text { como nódulos, quistes y } \\
\text { surcos después del cambio } \\
\text { de voz }\end{array}$ & $\begin{array}{l}12 \text { pacientes de } 13-17 \\
\text { años }\end{array}$ & $\begin{array}{l}\text { Retrospectivo. Revisión por larin- } \\
\text { gólogos de registros de videola- } \\
\text { ringoscopía y nasofibroscopía. } \\
\text { Cuestionario para evaluar molestias } \\
\text { vocales antes, durante y después } \\
\text { de la muda vocal }\end{array}$ & $\begin{array}{l}\text { Se demostró que luego de la muda } \\
\text { vocal: } \\
\text { - Disminuyen las molestias vocales } \\
\text { - Lesiones que sugerían nódulos: } \\
\text { tendían a desaparecer } \\
\text { - Lesiones que sugerían quistes, } \\
\text { progresaban a sulcus } \\
\text { - Los pacientes que mantuvieron } \\
\text { molestias vocales luego de la } \\
\text { muda, tenían mayor rigidez cordal } \\
\text { antes de la muda vocal }\end{array}$ \\
\hline $\begin{array}{l}\text { Chernobel'sky } \\
\text { SI } 22 \\
\text { / Rusia / } \\
2016\end{array}$ & $\begin{array}{l}\text { Detectar el inicio de la } \\
\text { muda vocal en niños } \\
\text { cantantes }\end{array}$ & $\begin{array}{l}30 \text { cantantes, divididos en } \\
2 \text { grupos. } \\
\text { Grupo A: } 11 \text { años } 10 \\
\text { meses - } 12 \text { años cuatro } \\
\text { meses } \\
\text { Grupo B: } 12 \text { años y } 10 \text { me- } \\
\text { ses - } 13 \text { años dos meses }\end{array}$ & $\begin{array}{l}\text { Se realizó una prueba acústica y } \\
\text { laringoscopía }\end{array}$ & $\begin{array}{l}\text { Se demostró que la frecuencia funda- } \\
\text { mental en los niños del Grupo B fue } \\
\text { significativamente menor que en el } \\
\text { grupo A. La diferencia ascendió a dos } \\
\text { medios tonos y podría considerarse } \\
\text { como el inicio de la mutación vocal }\end{array}$ \\
\hline $\begin{array}{l}\text { Balasubrama- } \\
\text { niam } \\
\text { R y col.23 } \\
\text { / India / } 2017\end{array}$ & $\begin{array}{l}\text { Investigar la edad de logro } \\
\text { de la mutación vocal en } \\
\text { niños y niñas }\end{array}$ & $\begin{array}{l}600 \text { participantes de } 8-18 \\
\text { años } \\
10 \text { grupos con un intervalo } \\
\text { de un año }\end{array}$ & $\begin{array}{l}\text { Mediante fonación sostenida y una } \\
\text { narración se comparó la frecuencia } \\
\text { fundamental y frecuencia de forman- } \\
\text { tes, por año y sexo }\end{array}$ & $\begin{array}{l}\text { 1. Con un corte de } 140 \mathrm{~Hz} \text { en niños } \\
\text { y } 240 \mathrm{~Hz} \text { para niña, se determinó } \\
\text { que a los } 16 \text { y } 15 \text { años, inicia la } \\
\text { mutación vocal en niños y niñas, } \\
\text { respectivamente 2. La frecuencia } \\
\text { del primer formante sufre cambios } \\
\text { a partir de los } 13 \text { años }\end{array}$ \\
\hline $\begin{array}{l}\text { Damasceno L } \\
\text { y col. } 24 \\
\text { / Brasil / } \\
2018\end{array}$ & $\begin{array}{l}\text { Analizar la relación entre } \\
\text { autopercepción vocal y } \\
\text { el impacto psíquico en } \\
\text { adolescentes, durante su } \\
\text { muda vocal }\end{array}$ & $\begin{array}{l}\text { Seis adolescentes de } \\
13 \text { años y } 5 \text { meses a } 14 \\
\text { años y } 11 \text { meses, que } \\
\text { cursaban el octavo año de } \\
\text { educación }\end{array}$ & $\begin{array}{l}\text { Evaluación perceptivo-auditiva de } \\
\text { la voz } \\
\text { Análisis cualitativo con un grupo } \\
\text { focal }\end{array}$ & $\begin{array}{l}\text { 1. Los sujetos describieron extrañeza } \\
\text { y preocupación por los cambios de } \\
\text { la adolescencia, sobre todo las re- } \\
\text { lacionadas con trastornos de la voz zo } \\
\text { 2. Señalan que el proceso de cambio } \\
\text { de voz es un factor negativo para } \\
\text { la interacción con sus interlocuto- } \\
\text { res, con repercusión en imagen } \\
\text { corporal y en identidad }\end{array}$ \\
\hline $\begin{array}{l}\text { Oliveira y } \\
\text { col. }^{26}{ }^{2} \\
\text { / Brasil// } \\
2018\end{array}$ & $\begin{array}{l}\text { Desarrollar un programa } \\
\text { de capacitación sobre la } \\
\text { muda vocal y hábitos vo- } \\
\text { cales saludables: Proyecto } \\
\text { Young Doctor }\end{array}$ & $\begin{array}{l}11 \text { estudiantes de secun- } \\
\text { daria: nueve niñas y dos } \\
\text { niños, de } 13 \text { a } 15 \text { años a } \\
\text { quienes se denominaron: } \\
\text { "médicos jóvenes" }\end{array}$ & $\begin{array}{l}\text { Se desarrolló un programa de } \\
\text { aprendizaje virtual, para capacitar a } \\
\text { los estudiantes } \\
\text { Todos los participantes respondie- } \\
\text { ron un cuestionario antes y después } \\
\text { del programa }\end{array}$ & $\begin{array}{l}\text { 1. Hubo aumento de conocimiento de } \\
\text { temas del curso } \\
\text { 2. Se observó que los médicos } \\
\text { jóvenes compartieron lo aprendido } \\
\text { a otros estudiantes de la escuela }\end{array}$ \\
\hline
\end{tabular}


esta etapa de desarrollo. El objetivo es controlar los sentimientos de incertidumbre y descontento, así como fomentar hábitos saludables durante este frágil periodo. ${ }^{26}$

\section{DISFONÍA INFANTIL Y MUDA VOCAL}

Existe una creencia que la disfonía infantil mejora luego de la muda vocal. En la investigación de Santos y colaboradores se demuestra que, efectivamente, posterior a la muda vocal la mayoría de los pacientes con disfonía disminuyen las molestias vocales, mientras que ciertas lesiones estructurales tienden a desaparecer o progresar. Los sujetos que no mejoraron fueron aquéllos que presentaban rigidez cordal premuda vocal. ${ }^{27}$

\section{DISCUSIÓN}

La voz es un medio de comunicación sumamente valioso que nos da identidad y nos distingue de otras personas. El periodo de muda vocal es un proceso natural en la vida de los adolescentes de ambos sexos, que otorga una nueva voz y, por ende, una nueva imagen personal.

La evaluación de la muda vocal se debe realizar mediante un equipo interdisciplinario que logre objetivar la anatomía y funcionalidad de las cuerdas vocales y realizar un completo análisis de los parámetros de la voz. Asimismo, complementar dicha evaluación con la valoración de los aspectos emocionales que se pueden desencadenar.

Si bien, es un periodo corto y que muchas veces pasa desapercibido, debería educarse a la población en etapa de pubertad, lo mismo que a docentes, profesores de canto, médicos y terapeutas de la voz, para estar atentos a identificar problemas. El proceso de capacitación debería incluir el conocimiento sobre la edad en que ocurre, tiempo de duración y las características vocales que se esperan en una muda vocal normal, tanto en hombres como en mujeres.

Un aspecto a señalar es que, en la búsqueda de la información, no se encontraron estudios longitudinales en poblaciones con y sin alteraciones vocales. Lo mismo fue observar que hay poca información acerca del periodo de muda vocal en mujeres.

Por último, consideramos que resulta fundamental informar y acompañar a los sujetos que están experimentando un desarrollo de cambio vocal para que éste se realice de la manera más fisiológica posible, para evitar consecuencias perjudiciales a nivel del aparato vocal o en el ámbito emocional.

\section{BIBLIOGRAFÍA}

1. Guimarães MF, Behlau MS, Panhoca I. Análise perceptivoauditiva da estabilidade vocal de adolescentes em diferentes tarefas fonatórias. Pró-Fono R Atual Cient. 2010; 22(4): 455458.

2. Anelli W, Costa H, Duprat A, Eckley C. Entendendo a muda vocal. Laringologia pediátrica. Sao Paulo: Roca; 1999. pp. 39-44.

3. Behlau M. Voz: o livro do especialista. Rio Janeiro: Revinter; 2005.

4. Marinho A. Disfonias e alterações hormonais. In: Costa HO, Duprat AC, Eckey CA. Laringologia pediátrica. Sao Paulo: Roca; 1999. pp. 23-38.

5. Neto P. Muda vocal refletindo sobre a imagem vocal do adolescente Rio de Janeiro. 1999.

6. Aronson AE, Bless D. Clinical voice disorders. New York Thieme; 2011.

7. Behlau M. Avaliação e tratamento das disfonias. Sao Paulo: Editora Lovise; 1995.

8. Fuchs M, Fröehlich M, Hentschel B, Stuermer IW, Kruse E, Knauft D. Predicting mutational change in the speaking voice of boys. $J$ Voice. 2007; 21(2): 169-178.

9. Alcantara de Oliveira Santos M, Pechula Moura JM, de Campos Duprat A, Olival Costa H, Benatti de Azevedo B. A interferência da muda vocal nas lesões estruturais das pregas vocais. Rev Bras Otorrinolaringol. 2007; 73(2): 226-230.

10. Dagli M, Sati I, Acar A, Stone RE Jr, Dursun G, Eryilmaz A. Mutational falsetto: intervention outcomes in 45 patients. J Laryngol Otol. 2008; 122(3): 277-281.

11. Andrews ML, Summers AC. Voice treatment for children and adolescents. San Diego, CA: United Nations Publications; 2002.

12. Cooksey J. Voice transformations in male adolescents. In: Welch $\mathrm{T}$, editor. Bodymind and voice: foundations of voice education. lowa City: The Voice Care Network; 2000.

13. Eliorriaga A. La continuidad del canto durante el periodo de muda vocal. Madrid: Universidad Autónoma de Madrid; 2011.

14. Boone DR, McFarlane SC, Von Berg SL, Zraick RI. The voice and voice therapy. Boston, MA: Pearson Higher Ed; 2013.

15. Piassi M. Etiología da Puberfonia. Sao Paulo: Cefac. Centro de Especializaçao em fonoaudiología clínica; 1998.

16. Morrison MD, Nichol H, Rammage L. The management of voice disorders. New York: Springer; 2013.

17. Wojciechowska A, Obrebowski A, Studzińska K, Swidziński P. Zaburzenia glosu mutacyjnego uwarunkowane czynnikami psychicznymi [Mutation voice disorders conditioned by psychic factors]. Otolaryngol Pol. 2010; 64(1): 51-54.

18. Pinho SMR. Tópicos em voz. Rio de Janeiro: Guanabara Koogan; 2001.

19. Urra-Barandiarán A, Vázquez-de-la-Iglesia F, FernandezGonzalez S, Molina M. Voz del niño. Rev Med Univ Navarra. 2006; 50 (3): 31-43.

20. Harris T. The voice clinic handbook. New York: Whurr; 2002.

21. Saida H, Okamoto M, Imaizumi S, Hirose H. A study of voice mutation and physical growth--a longitudinal observation. Nihon Jibiinkoka Gakkai kaiho. 1990; 93(4): 596-605.

22. Chernobel'sky SI. The acoustic changes of the voice in the singing boys during the permutation period. Vestn Otorinolaringol. 2016; 81 (2): 57-58. 
23. Balasubramaniam RK, N N. Voice mutation during adolescence in Mangalore, India: implications for the assessment and management of mutational voice disorders. J Voice. 2017; 31(4): 511.e29-511.e33.

24. Damasceno LL, de Andrada MA, Ghirardi ACdAM, Cunha MC. Relações entre autopercepção vocal e psiquismo em um grupo de adolescentes do sexo masculino na muda vocal. Distúrbios da Comunicação. 2018; 30(1): 117-127.
25. Gil D, Lourenço L, Miranda AR, Pereira AJ, Rodrigues S, Behlau M. A memória da muda vocal. Acta AWHO. 1994; 13(2): 74-80.

26. Oliveira LFd, Corrêa CdC, Vieira MMRM, Blasca WQ, Brasolotto AG. Intervention via teleducation about vocal mutation and vocal habits. Audiol Commun Res. 2018; 23: e1899.

27. Santos MA, Moura JM, Duprat Ade C, Costa HO, de Azevedo BB. The interference of voice change on structural vocal cords lesions. Braz J Otorhinolaryngol. 2007; 73(2): 226-230. 